\title{
Points rationnels sur les sous-groupes compacts des groupes algébriques
}

\author{
Daniel Bertrand
}

TABLE DES MATIÈRES

1. Propriété de densité et rigidité

2. Le cas des variétés semi-abéliennes

3. Extensions vectorielles et indépendance algébrique

Remerciements

Bibliographie
On étudie dans quelle mesure un point rationnel d'ordre infini $d^{\prime}$ une extension d'une variété abelienne par un groupe linéaire peut être situé sur son sous-groupe compact maximal (pour la topologie réelle). La théorie des formes modulaires permet de construire de tels points dans le cas torique, et cela fournit un contre-exemple a une propriété de densité récemment introduite par Waldschmidt. On démontre en revanche qu'il n'en existe pas sur l'extension vectorielle universelle d'une courbe elliptique.

We study to what extent a rational point of infinite order on an extension of an abelian variety by a linear group can lie in the extension's maximal compact subgroup (for the real topology). The theory of modular forms allows us to construct such points in the toric case, and this gives a counterexample to a density property recently introduced by Waldschmidt. In contrast, we show that there are no such points in the universal extension of an elliptic curve.

Soient $K$ un corps de nombres plongé dans le corps $\mathbb{R}$ des nombres réels, et $G$ un groupe algébrique commutatif connexe, défini sur $K$. Dans un article récemment paru dans cette revue, $M$. Waldschmidt [1994] étudie sous quelles conditions un ensemble de points $K$-rationnels de $G$ engendre topologiquement le groupe de Lie $G(\mathbb{R})$ des points réels de $G$. La propriété de densité qu'il introduit à ce propos quantifie, dans le cas des groupes, la conjecture de densité de Mazur [1992].

Comme il est expliqué dans [Waldschmidt 1994], cette propriété de densité est très probablement satisfaite quand $G$ est isogène au produit d'une variété abélienne $A$ par un groupe linéaire $L$. Le premier but du présent article est de montrer qu'elle ne l'est pas en général : notre contre-exemple, décrit 
au théorème 2.1 et à la proposition 2.2 , consiste en une extension d'une surface abélienne $A$, quotient de la jacobienne d'une courbe modulaire, par le groupe multiplicatif $L=\mathbb{G}_{m}$.

Dans la vérification que cette extension en fournit bien un contre-exemple, la propriété de densité de Waldschmidt n'intervient ici que par la conséquence qualitative suivante, établie au lemme 1.1, où on suppose $L$ déployé sur $K$, et les groupes algébriques $A$ et $L$ simples sur $K$ : si $G$ est une extension non isotriviale de $A$ par $L$ vérifiant la propriété de densité, alors il n'existe pas de points $K$-rationnels d'ordre infini sur le sous-groupe compact maximal $G^{c}$ du groupe de Lie $G(\mathbb{R})$.

Comme le laisse prévoir notre titre, c'est donc finalement de l'existence de points rationnels de ce type qu'il sera ici question. On en construit au $\S 2$ sur certaines variétés semi-abéliennes (et cela fournit le contre-exemple annoncé), tandis que le $\S 3$ est consacré aux extensions vectorielles, où tout porte au contraire à penser qu'il n'en existe pas. En guise de conclusion, on relie cette étude à des problèmes d'indépendance algébrique d'une part, et de hauteurs sur les variétés réglées d'autre part.

(À l'exception du lemme 1.1, la lecture du présent article ne nécessite ainsi pas de se reporter à celui de Waldschmidt. Nous renvoyons néanmoins le lecteur intéressé par l'énoncé précis de la propriété de densité à [Waldschmidt 1994, §1], où sa genèse est discutée en détail.)

\section{PROPRIÉTÉ DE DENSITÉ ET RIGIDITÉ}

Dans la catégorie des groupes algébriques sur $K$, tout groupe $G$ comme ci-dessus s'inscrit dans une suite exacte canonique

$$
0 \longrightarrow L \longrightarrow G \stackrel{\pi}{\longrightarrow} A \longrightarrow 0,
$$

où $L$ désigne le sous-groupe linéaire connexe maximal de $G$, et $A$ est une variété abélienne. On supposera ici $L$ déployé sur $K$, et on notera $H^{+}$la composante neutre d'un groupe de Lie $H$. Dans ces conditions, $L(\mathbb{R})^{+}$est isomorphe à un groupe vectoriel, et $G(\mathbb{R})^{+}$, extension de $A(\mathbb{R})^{+}$par un groupe isogène à $L(\mathbb{R})^{+}$, s'inscrit dans une nouvelle suite exacte

$$
0 \longrightarrow L(\mathbb{R})^{+} \longrightarrow G(\mathbb{R})^{+} \stackrel{\pi^{\prime}}{\longrightarrow} A^{\prime}(\mathbb{R}) \longrightarrow 0,
$$

où $A^{\prime}(\mathbb{R})$ est un tore réel, isogène à $A(\mathbb{R})^{+}$. Mais une telle extension est triviale dans la catégorie des groupes de Lie réels, de sorte que la projection $\pi^{\prime} \mathrm{y}$ admet une unique section $s$, d'image le sous-groupe compact maximal $G^{c}$ de $G(\mathbb{R})^{+}$. Celui-ci est donc ici isomorphe à $A(\mathbb{R})^{+}$.

Pour fixer les idées, nous supposerons de plus que $L$ et $A$ sont simples dans la catégorie des groupes algébriques sur $K$, et que l'extension $G$ n'y est pas isogène à une extension triviale. En particulier, $L=\mathbb{G}_{a}$ ou $\mathbb{G}_{m}$, et dans ce deuxième cas, l'extension $G$ correspond à un point $K$-rationnel $w=w(G)$ d'ordre infini sur la variété abélienne $A^{v}$ duale de $A$. Si $L(\mathbb{R})^{+}$reste simple dans la catégorie des groupes de Lie, cela n'est en général plus vrai de $A(\mathbb{R})^{+}$. Néanmoins, sous l'hypothèse que $A$ vérifie la propriété de densité, aucun des sousgroupes de Lie (fermés) propres de $A(\mathbb{R})^{+}$ne contient de point $K$-rationnel d'ordre infini. De même, on vient de voir que $G(\mathbb{R})^{+}$contient toujours strictement plus de sous-groupes de Lie (tel $G^{c}$ ) que $G$ ne contient de sous-groupes algébriques, mais on a :

Lemme 1.1. Soit $G$ une $K$-extension non isotriviale d'une variété abélienne A simple sur $K$ par $L=\mathbb{G}_{a}$ ou $\mathbb{G}_{m}$. Si $G$ satisfait la propriété de densité, tout point $K$-rationnel de $G$ situé sur $G^{c}$ est d'ordre fini.

Démonstration. Supposons qu'il existe un point $\gamma_{2}$ de $G(K) \cap G^{c}$ d'ordre infini, et reprenons les notations du début de ce paragraphe. Puisque $L(\mathbb{R})^{+}$ ne rencontre $G^{c}$ qu'en 0, le sous-groupe $\Gamma$ de $G(K)$ engendré par $\gamma_{2}$ et par un point d'ordre infini $\gamma_{1}$ de $L(K) \cap L(\mathbb{R})^{+}$est de rang 2 , et l'image $\pi(\Gamma)$ de $\Gamma$ dans $A$ est de rang 1 . Comme expliqué au corollaire 4.8(c) de [Waldschmidt 1994], on déduit sous ces hypothèses de la propriété de densité pour $G$ que $\Gamma$ est dense dans $G(\mathbb{R})^{+}$pour la topologie classique. En effet, l'adhérence de Zariski de $\mathbb{Z}\left(\gamma_{1}, \gamma_{2}\right)$ dans $G \times G$ est $H=L \times G$, puisque l'extension 
$G$ n'est pas isotriviale. Soient alors $\eta_{2}$ un élément de $G(\mathbb{R})^{+}$non situé sur $G^{c}$, dont l'image par $\pi^{\prime}$ engendre $A^{\prime}(\mathbb{R})$ pour la topologie classique, Log le logarithme sur $L(\mathbb{R})^{+}$, et $\eta_{1} \neq 0$ un point de $L(\mathbb{R})^{+}$ tel que 1 ,

$$
\frac{\log \left(\eta_{2}-s \pi^{\prime}\left(\eta_{2}\right)\right)}{\log \eta_{1}}
$$

et les coordonnées d'un logarithme de $\pi^{\prime}\left(\eta_{2}\right)$ dans une base des périodes de $A^{\prime}(\mathbb{R})$ soient linéairement indépendants sur $\mathbb{Z}$. D'après Kronecker, le groupe engendré par $\eta_{1}$ et $\eta_{2}$ dans $G(\mathbb{R})^{+}$est alors dense, tandis que le point $\left(\eta_{1}, \eta_{2}\right)$ de $G \times G$ appartient, comme requis pour cette propriété, à $H(\mathbb{R})$.

Pourtant, $\Gamma$ n'est pas dense dans $G(\mathbb{R})^{+}$, puisque son image $\mathbb{Z} \gamma_{1}$ par la rétraction $\operatorname{id}_{G}-s \pi^{\prime}$ de $G(\mathbb{R})^{+}$ sur $L(\mathbb{R})^{+}$a pour rang 1 et ne peut donc l'être dans $L(\mathbb{R})^{+}$. L'hypothèse faite sur $\gamma_{2}$ est ainsi contredite.

Remarque 1.2. Bien entendu, le lemme 1.1 ne s'étend pas aux produits $G$ d'une variété abélienne (non nulle) $A$ par un groupe linéaire. Il serait d'ailleurs aussi mis en défaut par les tores non déployés $G$, et pour la même raison: pour tout facteur anisotrope $A^{\prime}$ de $G$, le groupe $A^{\prime}(\mathbb{R})$ est compact. Ainsi, quand

$$
G=\mathrm{SO}_{2} / \mathbb{Q}
$$

$G(\mathbb{Q})=G^{c} \cap G(\mathbb{Q})$ correspond aux solutions rationnelles de l'équation $x^{2}+y^{2}=1$. Plus généralement, il existe des nombres algébriques, de degrés arbitrairement grands, dont toutes les valeurs absolues archimédiennes sont égales à 1 sans qu'ils soient des racines de l'unité.

Dans [Bertrand 1994; a] sont donnés deux types de construction de points rationnels sur les sousgroupes compacts maximaux d'extensions de variétés abéliennes par un tore. Tous deux sont liés à l'existence de couples de points orthogonaux pour l'accouplement de Néron-Tate sur les groupes de Mordell-Weil. C'est la construction de [Bertrand a] qui va ici servir.

\section{LE CAS DES VARIÉTÉS SEMI-ABÉLIENNES}

Théorème 2.1. Soit $(A, \lambda)$ une variété abélienne polarisée définie sur $K$, simple sur $K$, et admettant un $K$-endomorphisme non invariant sous l'involution de Rosati attachée à la polarisation $\lambda$. Si $A(K)$ est infini, il existe une $K$-extension non isotriviale $G$ de $A$ par $\mathbb{G}_{m}$, telle que $G^{c}$ contienne des points $K$-rationnels d'ordre infini.

Démonstration. Comme $A(K)$ est infini, il existe un point $K$-rationnel $w$ d'ordre infini sur la variété $A^{v}$ duale de $A$. Vu l'hypothèse faite sur les endomorphismes de $A$, on dispose par ailleurs d'un $K$-homomorphisme $h$ de $A^{v}$ vers $A$ distinct de son transposé $h^{v}$, et la simplicité de $A$ entraîne que le point $x=\left(h-h^{v}\right)(w)$ est d'ordre infini dans $A(K)$. Grâce à une construction de K. Ribet [Jacquinot et Ribet 1987], ce point $x$ se relève en un point $K$ rationnel canonique $\gamma=\gamma(w, h)$ de l'extension $G$ de $A$ par $\mathbb{G}_{m}$ paramétrée par $w$, et le théorème 4 de [Bertrand a] affirme que $\gamma$ appartient au sousgroupe compact maximal de $G(\mathbb{R})$. Or $\gamma$ est d'ordre infini dans $G(K)$, puisque $\pi(\gamma)=x$ l'est déjà dans $A(K)$.

En vertu du Lemme 1.1, une telle extension $G$ ne vérifie pas la propriété de densité. Pour en justifier l'existence, il nous reste toutefois à construire un corps de nombres réel $K$ et une variété abélienne $A$ sur $K$ vérifiant les hypothèses du théorème 2.1. C'est l'objet de l'énoncé suivant.

Proposition 2.2. Il existe un corps de nombres quartique totalement réel $K$ et une surface abélienne polarisée $(A, \lambda)$ définie sur $K$ et simple sur $K$, tels que l'involution de Rosati attachée à $\lambda$ agisse de façon non triviale sur $\operatorname{End}(A / K)$, et que le groupe de Mordell-Weil $A(K)$ soit infini.

La démonstration de cette proposition repose sur une construction classique de Shimura [1971, § 7.7 ; 1972, §10] (voir aussi [Ribet 1980]), intimement liée à l'existence de $\mathbb{Q}$-courbes elliptiques au sens de Ribet [1992] (voir aussi [Shimura 1972, p. 184]). Dans l'espoir de voir baisser le degré de $K$, nous 
rappelons tout d'abord le cadre général de cette construction.

Soit $N$ un nombre premier, congru à 1 modulo 4. Notons $\psi$ le symbole de Legendre relatif à $N$, et $f$ une forme modulaire parabolique normalisée de poids 2 , de niveau $N$ et de Nebentypus $\psi$, vecteur propre pour les opérateurs de Hecke. Shimura [1971, thórème 7.14] attache à $f$ et à ses compagnes une variété abélienne $A=A_{f}$ définie sur $\mathbb{Q}$, qu'on peut voir comme une sous-variété abélienne de la jacobienne de la courbe modulaire $X_{1}(N)$, et qui admet donc une polarisation $\lambda$ définie sur $\mathbb{Q}$. La $\mathbb{Q}$ sous-algèbre de $\operatorname{End}(A / \mathbb{Q}) \otimes \mathbb{Q}$ engendrée par les restrictions à $A$ des opérateurs de Hecke est isomorphe au corps de définition $E$ des coefficients de $f$, et coïncide en fait avec $\operatorname{End}(A / \mathbb{Q}) \otimes \mathbb{Q}$, en vertu de [Ribet 1980, corollaire 4.2] (d'après [Shimura 1972, remarque 1.7], $f$ n'est d'ailleurs pas de type CM). Enfin, $E$ est un corps CM (puisque $\psi$ est non trivial; voir [Shimura 1971, théorème 7.16]), et l'involution de Rosati définie par $\lambda$ induit sur $E$ la conjugaison complexe (d'après la positivité de la métrique de Weil). Ainsi, $A / \mathbb{Q}$ est une variété abélienne $\mathbb{Q}$-simple, qui admet des $\mathbb{Q}$-endomorphismes non nuls antisymétriques sous l'involution de Rosati : ça sera par exemple le cas des restrictions à $A$ des opérateurs de Hecke $T_{n, \psi}$ d'indices premiers non résidus quadratiques modulo $N$.

J'ignore si ce type de de variété abélienne peut présenter un rang de Mordell-Weil $>0$ sur $\mathbb{Q}$ (voir aussi la question 2.5 ci-dessous). Mais il est facile de lui attacher un corps de nombres $K$ remplissant les conditions de la proposition 2.2. Pour revenir à son énoncé, supposons $A$ de dimension 2, en choisissant par exemple $N=29$, qui donne $E=\mathbb{Q}(\sqrt{-5})$ [Shimura 1971, p. 207]. Si $k$ désigne le corps quadratique réel $\mathbb{Q}(\sqrt{N})$, et $s$ son automorphisme non trivial, $A$ est alors $k$-isogène au carré d'une courbe elliptique $C$ définie sur $k$, isogène sur $k$ à sa conjuguée $C^{\sigma}$ et n'admettant (même sur $\mathbb{C})$ pas de multiplication complexe. Plus précisément, $A$ est $\mathbb{Q}$-isogène à la variété abélienne $B$ déduite de $C$ par restriction des scalaires de $k$ à $\mathbb{Q}$ [Ribet 1992, $\S 7$; Shimura 1972, p. 180]. Dans ces conditions, si $K$ désigne un corps de nombres plongé dans $\mathbb{R}$ et linéairement disjoint de $k$, la $k$ algèbre $K \otimes_{\mathbb{Q}} k$ s'identifie au corps $K k=K(\sqrt{N})$, et $B(K)$ est isomorphe à $C\left(K \otimes_{\mathbb{Q}} k\right)=C(K \sqrt{N})$. Dès que $C(K \sqrt{N})$ est infini, $A(K)$ le sera aussi, et on conclura en notant que $A$ reste simple sur $K$, puisque toutes les sous-variétés abéliennes de $A / \mathbb{C}$ sont définies sur $k$.

Reste donc à prouver, en notant $p$ le nombre premier $N$ :

Lemme 2.3. Soit $C$ une courbe elliptique définie sur $\mathbb{Q}(\sqrt{p})$. Il existe un corps (quartique) totalement réel $K$ où $p$ n'est pas un carré, tel que $C(K(\sqrt{p}))$ soit infini.

\section{Démonstration. Soit}

$$
Y^{2}=X^{3}+\left(b+b^{\prime} \sqrt{p}\right) X+\left(c+c^{\prime} \sqrt{p}\right)
$$

une équation de Weierstrass de $C$ sur $\mathbb{Z}[\sqrt{p}]$. A tout entier rationnel $x$ suffisamment grand et tel que $x^{3}+b x+c$ soit modulo $p$ un carré $\neq 0$, nous allons attacher un corps totalement réel $K_{x}$ de degré $\leq 4$ où $p$ n'est pas carré, et deux éléments $y$ et $y^{\prime}$ de $K_{x}$ tel que le point

$$
(X, Y)=\left(x, y+y^{\prime} \sqrt{p}\right)
$$

appartienne à $C\left(K_{x}\right)$. Comme les points de torsion de $C$ définis sur un corps de degré $\leq 8$ sont en nombre fini, l'un de ces points $(X, Y)$ sera d'ordre infini, et le corps $K=K_{x}$ correspondant répondra à la question.

Fixons donc (une classe de congruence modulo $p$ de) $x$ comme ci-dessus ; il en existe par Hasse-Weil, même si $p$ divise $-4 b^{3}-27 c^{2}$. Alors, $\left(x, y+y^{\prime} \sqrt{p}\right)$ appartient à $C$ dès que

$$
\begin{aligned}
y^{2}+p y^{\prime 2} & =x^{3}+b x+c:=A(x), \\
2 y y^{\prime} & =b^{\prime} x+c^{\prime}:=2 B(x) ;
\end{aligned}
$$

donc, pour $B(x) \neq 0$ (le cas $B$ identiquement nul pouvant être traité directement), dès que

$$
f(y):=y^{4}-A(x) y^{2}+p B^{2}(x)=0 .
$$


Pour $x \gg 0$, on a $A^{2}(x)-4 p B^{2}(x)>0$, et $f$ admet quatre racines réelles. Par ailleurs, la congruence

$$
f(\psi) \equiv y^{2}\left(y^{2}-A(x)\right) \equiv 0 \bmod p
$$

admet, vu le choix de la classe $x$ modulo $p$, deux racines simples dans $\mathbb{F}_{p}$, donc $f(y)=0$ admet au moins deux racines $\pm y(x)$ dans $\mathbb{Q}_{p}$. Ainsi, $p$ est non ramifié dans le corps totalement réel $K_{x}=$ $\mathbb{Q}(y(x))$, et $p$ ne peut y être un carré, tandis que le point $(X, Y)=\left(x, y(x)+B(x) y(x)^{-1} \sqrt{p}\right)$ appartient bien à $C\left(K_{x}\right)$.

Remarque 2.4. Ce sont précisément les endomorphismes antisymétriques de $A_{f} / \mathbb{Q}$ qui permettent la construction dans [Shimura 1971, théorème 7.30; 1972, remarque 2.10] d'extensions abéliennes non cyclotomiques du corps quadratique réel $k$. Les unités abéliennes que [Bertrand a, remarque 5] propose d'étudier sont-elles liées à ces corps de classes?

La proposition 2.2 conduit à quelques questions naturelles. Tout d'abord, vaut-elle avec $K=\mathbb{Q}$ (sans imposer nécessairement que $A$ soit de dimension deux)? En particulier :

Question 2.5. Existe-t-il une forme nouvelle $f$ de poids 2 , de conducteur un nombre premier $N \equiv 1$ $\bmod 4$ et de caractère $\psi=(\cdot / N)$ telle que le rang de $A_{f}(\mathbb{Q})$ soit $\geq 1$ ?

La connaissance des valeurs en $s=1$ des fonctions $L(f, s)$ devrait fournir un élément de réponse, mais l'absence de signe dans l'équation fonctionnelle de $\zeta\left(A_{f} / \mathbb{Q}, s\right)$ n'en facilite pas l'étude. J. Cremona, consulté sur ce point, me signale qu'il est en fait plus simple (quand $[E: \mathbb{Q}]=2$ ) d'étudier directement les points $\mathbb{Q}(\sqrt{N})$-rationnels de la courbe elliptique $C$. Il a vérifié avec P. Serf qu'il n'en existe pas d'ordre infini pour $N=41,337$, ou 881, tandis qu'une première recherche sur $N=29$ et 37 n'a donné que des points d'ordre 3 et 5 , respectivement.

Supposons néanmoins qu'on puisse remplacer $K$ par $\mathbb{Q}$ dans la proposition 2.2 et soit $x$ un point d'ordre infini de $A(\mathbb{Q})$. Son image $w$ sous n'importe quel homomorphisme antisymétrique de $A$ vers $A^{v}$ est orthogonale à $x$ pour l'accouplement canonique de Néron-Tate attaché au fibré de Poincaré sur $A \times A^{v}$. Comme $\mathbb{Q}$ n'a qu'une place archimédienne, il est alors facile de relever $x$ en un point $\gamma_{2}$ de l'extension $G / \mathbb{Q}$ de $A$ par $\mathbb{G}_{m}$ paramétrée par $w$, tel que $\gamma_{2}$ soit situé sur le sous-groupe compact maximal de $G(\mathbb{R})$ [Bertrand 1994, $\S 3$.a], et de contredire ainsi directement la conclusion du lemme 1.1.

En second lieu, on peut se demander:

Question 2.6. Existe-t-il des variétés abéliennes polarisées $A / \mathbb{R}$ qui soient simples sur $\mathbb{C}$, mais qui admettent des $\mathbb{R}$-endomorphismes non invariants sous l'involution de Rosati?

Le résultat suivant montre que leur dimension sera nécessairement $\geq 4$.

Proposition 2.7. Soit $(A, \lambda)$ une surface abélienne polarisée définie sur $\mathbb{R}$. Si $A$ est simple sur $\mathbb{C}$, l'involution de Rosati attachée à $\lambda$ agit trivialement sur $\operatorname{End}(A / \mathbb{R})$.

Démonstration. Soit $D$ le corps des fractions de l'anneau des $\mathbb{C}$-endomorphismes de $A$. L'énoncé est trivial si $D$ est de type I au sens de la classification d'Albert. Par ailleurs, pour une $\mathbb{C}$-surface abélienne simple, $D$ ne peut être de type III (voir par exemple [Lange and Birkenhake 1992, 5.5.5]), et si $D$ est de type IV, c'est un corps CM ; ses multiplications complexes non totalement réelles n'agissent pas sur le réseau des périodes réelles, donc ne sont pas définies sur $\mathbb{R}$. Reste le cas (type II) où $D$ est une algèbre de quaternions indéfinie sur son centre (qui ne peut être que $\mathbb{Q}$ ). Alors, $(A, \lambda)$ fournit un point réel d'une courbe de Shimura, quotient du demi-plan de Poincaré $\mathcal{H}$ par un sous-groupe de congruence $\Delta$ du groupe des unités $D^{*}$ de $D$. Mais d'après [Shimura 1975, théorème 0], de telles courbes $\mathcal{H} / \Delta$ n'ont pas de points réels.

\section{EXTENSIONS VECTORIELLES ET INDÉPENDANCE ALGÉBRIQUE}

Le lemme 1.1 vaut que $L$ soit le groupe multiplicatif ou le groupe additif. Nous montrons maintenant que la situation est plus favorable dans ce 
deuxième cas. Rappelons que le corps de nombres $K$ est plongé dans $\mathbb{R}$.

Théorème 3.1. Soit $G$ une $K$-extension non triviale d'une courbe elliptique A par $\mathbb{G}_{a}$. Alors, tout point $K$-rationnel de $G$ situé sur le sous-groupe compact maximal $G^{c}$ de $G(\mathbb{R})$ est d'ordre fini. Plus généralement, tout sous-groupe $\Gamma$ de $G(K)$ de rang 2 , d'image $\pi(\Gamma)$ de rang 1 dans $A(K)$, est dense dans $G(\mathbb{R})^{+}$pour la topologie classique.

Démonstration. La preuve s'appuie sur des énoncés de transcendance, dûs à Waldschmidt et à Wüstholz, sur les périodes d'intégrales elliptiques de troisième espèce. Soient $\wp$ une fonction elliptique de Weierstrass d'invariants algébriques réels paramétrant $A(\mathbb{C}), \omega$ sa période réelle, $\eta$ la quasipériode de la fonction $\zeta$ de Weierstrass associée, et $u$ un logarithme réel d'un point $x$ de $A(K)$. Dire qu'un relevé $\gamma$ de $x$ à $G(K)$ appartient à $G^{c}$ équivaut à dire qu'il existe un élément $\alpha$ de $K$ tel que le point $(\zeta(u)-\alpha, u)$ soit situé sur la droite réelle engendrée dans $\mathbb{R}^{2}$ par la période $(\eta, \omega)$ de $G(\mathbb{R})$. En calculant le déterminant, on obtient

$$
\zeta(u) \omega-\eta u-\alpha \omega=0 .
$$

On reconnaît ici l'intégrale sur un cycle non trivial d'une forme différentielle de troisième espèce sur $A$, définie sur $K$, de diviseur résidu $(x)-(-x)$. D'après [Waldschmidt 1979, corollaire 3.2.12] (ou plus généralement, d'après le théorème de Wüstholz [1984] sur la méthode de Baker), une telle période ne peut être nulle que si $x$ est d'ordre fini dans $A(K)$. L'image sous l'exponentielle de

$$
(\zeta(u)-\alpha, u)=((\eta / \omega) u, u)
$$

est alors bien un point $\gamma$ d'ordre fini dans $G$.

De même, l'ensemble des logarithmes des éléments d'un sous-groupe $\Gamma$ vérifiant les conditions $\mathrm{du}$ théorème 2.1 est représentable par le groupe engendré par les vecteurs

$$
\{(\beta, 0),(\zeta(u)-\alpha, u),(\eta, \omega)\}
$$

dans $\mathbb{R}^{2}$, où $u$ et $\alpha$ sont comme ci-dessus, et $\beta$ est un nombre non nul de $K$. En écrivant les mineurs d'ordre 2 , on voit que $\Gamma$ ne peut éviter d'être dense dans $G(\mathbb{R})^{+}$que si les nombres

$$
\lambda_{u}(\omega):=\zeta(u) \omega-\eta u, \quad \omega, \quad u
$$

sont linéairement dépendants sur $K$. Mais vu l'hypothèse faite sur $\pi(\Gamma)$, les nombres $u$ et $\omega$ sont linéairement indépendants sur $\mathbb{Q}$, et le résultat de [Wüstholz 1984] fournit encore la conclusion recherchée.

Le cas des sous-groupes $\Gamma$ de rang 2 de $G(K)$ ne rencontrant $\mathbb{G}_{a}$ qu'en 0 conduit à une question actuellement ouverte d'indépendance quadratique de périodes de formes de troisième espèce, de type « problème des quatre exponentielles ». Plus précisément, établir la densité d'un tel sous-groupe dans $G(\mathbb{R})^{+}$équivaut à résoudre le problème suivant :

Question 3.2. Soient $\alpha$ et $\beta$ des nombres algébriques réels, et, avec les notations précédentes, $u$ et $v$ deux nombres réels où $\wp$ prend des valeurs algébriques, tels que $u, v$ et $\omega$ soient linéairement indépendants sur $\mathbb{Q}$. A-t-on alors:

$$
\frac{\lambda_{u}(\omega)-\alpha \omega}{u} \neq \frac{\lambda_{v}(\omega)-\beta \omega}{v} ?
$$

La conjecture d'indépendance algébrique proposée dans [Bertrand 1988, conjecture 2.b] fournirait une réponse positive à cette question (en vertu de [Bertrand 1988, p. 50, cas (v)]).

L'analogue du théorème 3.1 quand $A$ est une variété abélienne générale n'est non plus pas connu, et il serait intéressant de lui appliquer cette conjecture. La situation exceptionnelle rencontrée au théorème 2.1 reflète d'ailleurs les «relations de type Riemann » mentionnées dans [Bertrand 1988, page 50, cas (vi)]. La partie unipotente du groupe de Mumford-Tate du 1-motif attaché à cette situation [Bertrand a, §3.3] n'est plus gouvernée par le groupe algébrique $H$ de la propriété de densité, et c'est probablement là la raison profonde de sa mise en défaut.

Remarque 3.3. Comme il est expliqué dans [Bertrand a, $\S 3]$, le point $\gamma$ utilisé dans la preuve du 
théorème 2.1 appartient en fait au sous-groupe compact maximal de $G\left(K_{v}\right)$ pour tout complété $K_{v}$ de $K$, ce qui entraîne l'existence d'une infinité de points de $G(K)$ de «hauteur relative» nulle. Le théorème 3.1 , joint aux définitions et aux calculs de [Chambert-Loir], montre en revanche qu'aucun point d'ordre infini d'une extension non triviale d'une courbe elliptique par $\mathbb{G}_{a}$ n'est de « hauteur relative » nulle. Il serait intéressant d'étendre ces énoncés aux surfaces réglées et aux fibrés projectifs sur les variétés abéliennes, tout en les quantifiant.

\section{REMERCIEMENTS}

Je remercie L. Mérel, M. Waldschmidt et le referee pour leurs remarques sur ce travail, ainsi que $\mathrm{S}$. Levy pour son aide dans la préparation de l'article.

\section{BIBLIOGRAPHIE}

[Bertrand 1988] D. Bertrand, "Galois representations and transcendental numbers", pp. 37-55 dans New Advances in Transcendence Theory (édité par A. Baker), Cambridge Univ. Press, Cambridge, 1988.

[Bertrand 1994] D. Bertrand: "1-motifs et relations d'orthogonalité dans les groupes de Mordell-Weil", prépublication Univ. Paris VI, 1994.

[Bertrand a] D. Bertrand, "Minimal heights and polarizations on group varieties", à paraître dans le Duke Math. J.

[Chambert-Loir] A. Chambert-Loir, "Extension universelle de variétés abéliennes et hauteurs des points de torsion", à paraître dans Compositio math.

[Jacquinot et Ribet 1987] O. Jacquinot et K. Ribet, "Deficient points on extensions of abelian varities by $\mathbb{G}_{m} "$, J. Number Th. 25 (1987), 133-151.
[Lange and Birkenhake 1992] H. Lange et C. Birkenhake, Complex Abelian Varieties, Grundlehren der mathematischen Wissenschaften 302, Springer, Berlin, 1992.

[Mazur 1992] B. Mazur, "The topology of rational points", Experimental Math. 1 (1992), 35-45.

[Ribet 1980] K. Ribet, "Twists of modular forms and endomorphisms of abelian varieties", Math. Ann. 253 (1980), 43-62.

[Ribet 1992] K. Ribet, "Abelian varieties over $\mathbb{Q}$ and modular forms", pp. 53-79 dans Algebra and topology 1992, Taejŏn (édité par S. G. Hahn et D. Y. Suh), Korea Adv. Inst. Sci. Tech., Taejŏn, 1992. TEX source available at ftp ://math.berkeley.edu/pub/Preprints/ Ken_Ribet/korea.tex.

[Shimura 1971] G. Shimura, "Introduction to the Arithmetic Theory of Automorphic Functions", Iwanami Shoten, Tokyo, and Princeton Univ. Press, Princeton, 1971.

[Shimura 1972] G. Shimura, "Class fields over real quadratic fields and Hecke operators", Ann. Math. 95 (1972), 131-190.

[Shimura 1975] G. Shimura, "On the real points of an arithmetic quotient of a bounded symmetric domain", Math. Ann. 215 (1975), 135-164.

[Waldschmidt 1979] M. Waldschmidt, Nombres transcendants et groupes algébriques, Astérisque 69/70, Société Mathématique de France, Paris, 1979.

[Waldschmidt 1994] M. Waldschmidt, "Densité de points rationnels sur un groupe algébrique", Experimental Math. 3 (1994), 329-352.

[Wüstholz 1984] G. Wüstholz, "Recent progress in transcendence theory", pp. 280-296 dans Number theory / Journées arithmétiques, Noordwijkerhout, 1983 (édité par H. Jager), Lecture notes in mathematics 1068, Springer, Berlin, 1984.

Daniel Bertrand, Université Pierre et Marie Curie (Paris VI), Mathématiques, T. 46, Case 247, 4, Place Jussieu, F-75252 Paris Cédex 05, France (bertrand@mathp6.jussieu.fr)

Received Februrary 4, 1995 ; accepted June 4, 1995 